\title{
Using two-level stable storage for efficient checkpointing
}

\author{
L.M. Silva \\ J.G.Silva
}

Indexing terms: Checkpointing, Fault tolerance, Parallel computing systems

\begin{abstract}
Checkpointing and rollback recovery is a very effective technique to tolerate the occurrence of failures. Usually, checkpoint data is saved on disk, however, in some situations the time to write the data to disk can represent a considerable performance overhead. Alternative solutions would make use of main memory to maintain the checkpoint data. The paper starts by presenting two main memory checkpointing schemes: neighbour based and parity checkpointing. Both schemes have been implemented and evaluated in a commercial parallel machine. The results show that neighbour based checkpointing presents a very low performance overhead and assures a fast recovery for partial failures. However, it is not able to tolerate multiple and total failures of the system. To solve this shortcoming the authors propose a two-level stable storage integrating the use of neighbour based with disk based checkpointing. This approach combines the advantages of the two schemes: the efficiency of diskless checkpointing with the high reliability of disk based checkpointing.
\end{abstract}

\section{Introduction}

The mean time between failures (MTBF) of a parallel machine is considerably lower than a normal workstation, which increases the probability of failures and preventive shutdowns occurring during the execution of a parallel program. Some fault tolerance support is thereby required. Checkpointing allows long-running applications to save their state at regular intervals so that they may be restarted after interruptions without unduly retarding their progress. It is a feasible technique to tolerate transient failures and to avoid total loss of work. Each checkpoint is saved in some medium that is designated by stable storage [1]. By definition, the stable storage should be resilient to hardware crashes and software failures and should be immune to the phenomenon of memory decay. The write operations in stable storage should be atomic to the occur-

(C) IEE, 1998

IEE Proceedings online no. 19982440

Paper first received 20th July and in revised form 3rd November 1998

The authors are with Departamento Engenharia Informática, Universidade de Coimbra - POLO II, 3030 Coimbra, Portugal rence of failures. This means that every write operation is made completely or not at all; partial writes are not allowed to occur.

Usually, stable storage is implemented on disk. The main advantages of this approach are the smplicity, the increased level of reliability and the portaljility. To tolerate system crashes two checkpoint files hive to be maintained: the last established file and the working checkpoint file. If there is a failure while satving the new checkpoint, there is always the chance to recover using the old established checkpoint file. There are several ways to implement stable storage on disk [1-4].

Disk based stable storage is the approach generally used. However, in some applications that need to be checkpointed very frequently the use of the disk may result in a serious performance bottleneck. In fact, several experimental studies [5-8] have shown that the main source of performance overhead is the time used in writing the checkpoints to disk.

For this reason, some researchers have developed alternative solutions for stable storage based on the use of RAM. The schemes [9-11] use special memory boards or additional hardware mechanisms to prevent erroneous accesses to stable storage. These RAM based stable storage schemes provide much faster aciess than those schemes that made use of the disk. Unfortunately, they require changes in hardware, undermining their portability across commercial computing systems. An alternative solution is to use the available memory from other processors to save the checkpoint data. This approach would not require additional hardv/are and can be implemented in any parallel machine orovided there is enough spare memory among the different processors. It is not as reliable as disk based stable storage since it cannot tolerate the occurrence of a global failure of the machine. Its main use would be to tolerate single processor failures. At first sizht, this approach for stable storage is faster than using the disk.

The goal of our study was to evaluate the fisasibility of this approach for implementing stable storage in parallel machines and to compare the latency and the access bandwidth with a disk based stable storage approach. Finally, we integrate both stable storage approaches (diskless and disk based) and achieve a mixed solution that provides the best of these approaches: the efficiency of main memory checkpointing and the reliability of disk based stable storage. All the schemes described in this paper assume a crash failure model. Byzantine failures are not covered by these techniques. 


\section{Neighbour based checkpointing}

A technique that avoids checkpoint writing to disk is to use the main memory of neighbour processors. Processors of the network are organised in a virtual ring. Each processor saves its checkpoint into its physical memory (snapshot area) and into the neighbour processor that follows on the ring. The degree of replication is only one $(k=1)$, thus the scheme can tolerate only single failures. In practice it is able to tolerate more than one failure provided the failures do not occur in adjacent processors on the virtual ring.

This scheme is not robust against failures that occur during the checkpointing protocol. To tolerate these failures each processor has to allocate two checkpoint areas in its physical memory: one to keep its own checkpoint and another to maintain the checkpoint of its preceding neighbour. The first step is to save the application into the local snapshot area of each processor. Then, it sends the checkpoint to the next processor on the ring. During the first step the application process is blocked, while the second step can be done concurrently with the computation. At the end of each checkpoint operation the system swaps the identity of the memory areas. The extra memory space that is required by neighbour based checkpointing can be considerable since it represents twice the size of the application's state.

This neighbour based checkpointing scheme should not be used alone, since it is not able to recover from total failures of the system. In our opinion it would be more interesting to integrate this approach with a disk based checkpointing scheme. Thus, from time to time the system should take a global checkpoint to disk (we call these 'hard' checkpoints), and in between, the application can be checkpointed in a distributed way to processor memory (these are called 'soft' checkpoints). Assuming this hybrid approach it is possible to checkpoint the application more often, and the application is able to tolerate single failures with a minor overhead and total failures with a higher recovery latency. If there is a failure during the neighbour based checkpointing protocol and it is not possible to recover from the 'soft' checkpoint then the application can be restarted from the previous 'hard' checkpoint, that is kept on disk.

We have implemented this simple neighbour based scheme and a parity based checkpointing that will be described in the next Section.

\section{Parity based checkpointing}

Another possible way to implement diskless checkpointing is to use a parity based approach. This was originally proposed in [12] and evolves from the use of parity schemes in the development of reliable disk arrays [13]. In our case, it is not used to provide disk reliability, rather, it is used as a compressed way to save distributed checkpoints in the main memory of the processors. The basic idea is to avoid disk writing and to maintain enough redundant information about the checkpoint data to enable it to tolerate a single processor failure. As a result, the application should be able to checkpoint far more frequently than when checkpoints are saved on disk.

To tolerate one single failure we should use a $(N+1)$ parity technique. One processor in the network, the parity processor (PP), keeps a parity checkpoint of each global distributed checkpoint that is taken by the application. Each of the other processors saves its checkpoint into a local snapshot area. The checkpoint size of processor $P_{i}$ is $S_{i}$. This means that every processor should have an amount of unused memory at least of the same size as the local checkpoint.

After this local operation, all the checkpoint contents are XORed and saved in the parity checkpoint. The size of the parity checkpoint is calculated as:

$$
S_{\text {parity_chkp }}=\max \left(S_{i}\right), \quad i=0, \ldots, N-1
$$

The parity checkpoint is computed using the XOR operator. Let us assume that $b_{i j}$ corresponds to the $j$ th byte of $P_{i}$ 's checkpoint. If $j$ is higher than $S_{i}$ (but lower than $S_{\text {parity chkp }}$ ) then it is set to 0 . Then each byte of the parity checkpoint $(B)$ is computed in the following way:

$$
B_{j}=b_{1 j} \oplus b_{2 j} \oplus \cdots \oplus b_{n j} ; \quad 1 \leq j \leq S_{\text {parity_chkp }}
$$

This checkpoint is then saved on that parity processor, while every other processor maintains a copy of its own checkpoint. If a processor $P_{i}$ fails, the application can be recovered from the previous checkpoint. All the nonfailed processors restore their state from their local checkpoints, while the checkpoint of $P_{i}$ can be retrieved from all the others and the parity checkpoint, in the following way:

$b_{i j}=b_{1 j} \oplus \cdots \oplus b_{i-1 j} \oplus b_{i+1 j} \oplus \cdots \oplus b_{n j} \oplus B_{j} ; 1 \leq j \leq S_{i}$

If the parity processor fails then it can restore its state from the backup copy (kept on disk or in main memory) or by recalculating the parity checkpoint from scratch. We have used a basic scheme with one checkpoint per processor and one parity checkpoint. Although this scheme does not assure checkpoint atomicity it requires the minimum amount of extra memory.

\section{The Parix CHK-LIB}

Previous parity and neighbour based checkpointing schemes were implemented in a checkpointing library, CHK-LIB. CHK-LIB is a system library that runs on top of the Parix Operating System [14]. It works primarily as a communication library and provides support for checkpointing. Any user that is not interested in the fault tolerance facilities can use CHK-LIB as a normal communication library instead of using the Parix system interface. The programming interface of CHK-LIB was inspired by the MPI standard [15] to facilitate the porting of existing MPI programs to Parix. However, it was not a full implementation of MPI: only a small subset of the numerous MPI routines can be found in CHK-LIB.

The library implements several checkpointing and message logging mechanisms. It was not meant to be a commercial or production tool; rather it was developed to provide support for our study into checkpointing in parallel systems available in our department (i.e. Parsytec machines).

The use of parity and neighbour based schemes requires a semitransparent approach having the programming interface presented in Fig. 1. The CHK_Pack_chkp () routine is used to specify the critical data of the application. The checkpoint routine, CHK_Checkpoint(), saves the relevant application data, that is, those variables and data structures indicated by the programmer through use of the previous routine. The 
placement of checkpoints is under control of the user: he/she can make use of the global synchronisation points already existing in the application. It is the programmer's responsibility to place the checkpoint routines at points of the application that correspond to a consistent global state. Finally, the CHK_Restart() routine is used at the beginning of the application: if it is a restart from a previous checkpoint the program can skip the initialisation part, since the library will restore the values of the critical data structure from the last checkpoint.

int CHK_Pack_chkp (void *ptr, int size)

int CHK Restart (void);

int CHK_Checkpoint (void);

Fig. 1 Fault tolerant primitives of the CHK-LIB

\section{Implementation results}

In our experiments, we used a Xplorer Parsytec machine with eight transputers (T805). Each processor had 4 Mbytes of main memory. All the processors can read and write directly to the file system of the host machine, a Sun Sparc 2 Workstation. This I/O system may introduce a bottleneck during the checkpoint operation, but each processor was able to write into a different file without requiring collective synchronisation.

\subsection{Applications}

To evaluate the checkpointing schemes we used the following application benchmarks:

- ISING: this program simulates the behaviour of spin-glasses.

- SOR: successive overrelaxation is an iterative method to solve Laplace's equation on a regular grid.

- ASP: solves the all-pairs shortest paths problem.

- GAUSS: solves a system of linear equations using the method of Gauss elimination.

- NBODY: this program simulates the evolution of a system of bodies under the influence of gravitational forces.

\subsection{Parity versus neighbour based checkpointing}

In this Section we compare these two checkpointing approaches and evaluate their performance overhead. Neighbour based checkpointing is termed NBC, while parity checkpointing approach is termed $\mathbf{P B C}$.

The NBC technique, with a single degree of replication $(k=1)$, is able to tolerate single processor failures. In some cases it can tolerate more than one failure provided they occur in nonadjacent processors of the virtual ring. On the other hand, the PBC approach is able to tolerate only single processor failures. In order to tolerate total or multiple failures $\mathrm{PBC}$ or NBC schemes should be integrated with a disk based checkpointing mechanism. This two-level stable storage approach is described in the next subsection.

If we compare the two approaches, we can say that PBC always presents a lower memory overhead than NBC. However, it remains to be seen what the performance overhead is of both approaches. Next, we present a quantitative comparison between the NBC and $\mathrm{PBC}$ techniques. Five applications have been used and the overhead per checkpoint is presented in Table 1 .

Table 1: Overhead per checkpoint in seconds (NBC versus PBC)

\begin{tabular}{|c|c|c|c|c|}
\hline Application & $\begin{array}{l}\text { Size of } \\
\text { Chkp } \\
\text { (Kbytes) }\end{array}$ & $\begin{array}{l}\text { Overhead } \\
\text { per Chkp } \\
\text { NBC }\end{array}$ & $\begin{array}{l}\text { Overhead } \\
\text { per Chkf. } \\
\text { PBC }\end{array}$ & $\begin{array}{l}\mathrm{PBC} / \\
\mathrm{NBC}\end{array}$ \\
\hline SOR $256 \times 256$ & 540 & 0.123 & 2.923 & 23.7 \\
\hline SOR $512 \times 512$ & 2104 & 0.832 & 12.625 & 15.1 \\
\hline SOR $768 \times 768$ & 4692 & 2.207 & 29.108 & 13.1 \\
\hline SOR $1024 \times 1024$ & 8304 & 3.761 & 52.008 & 13.8 \\
\hline ISING $256 \times 256$ & 269 & 0.050 & 1.430 & 28.6 \\
\hline ISING $512 \times 512$ & 1049 & 0.111 & 5.497 & 49.5 \\
\hline ISING $768 \times 768$ & 2341 & 0.156 & 12.216 & 78.3 \\
\hline ISING $1024 \times 1024$ & 4145 & 0.430 & 21.711 & 50.4 \\
\hline ISING $1280 \times 1280$ & 6461 & 0.670 & 34.216 & 51.0 \\
\hline ASP 512 & 1024 & 0.202 & 5.820 & 28.8 \\
\hline ASP 1024 & 4096 & 0.584 & 24.298 & 41.6 \\
\hline GAUSS 512 & 2052 & 0.437 & 10.065 & 23.0 \\
\hline GAUSS 1024 & 8200 & 1.447 & 44.247 & 30.5 \\
\hline NBODY 4000 & 312 & 0.057 & 1.817 & 31.8 \\
\hline
\end{tabular}

The overhead per checkpoint is presented in seconds. As can be seen, the overhead introduced by the parity checkpointing scheme is much higher than that incurred by neighbour based checkpointing. The last column represents the relationship between the overhead of $\mathrm{PBC}$ over NBC. If we take the mean average, we can see that parity checkpointing perform; 34 times worse than neighbour based checkpointing.

Parity checkpointing performed even worse than disk based checkpointing. In Fig. 2 we present a comparison between PBC, NBC and disk based checkpointing (DBC). This last scheme uses the central disk to save checkpoint data but the remote write operations are done concurrently with the execution of the application. The state of the application is first saved into a memory buffer; after that, there is a checkpointer thread that sends this buffer to a remote disk file.

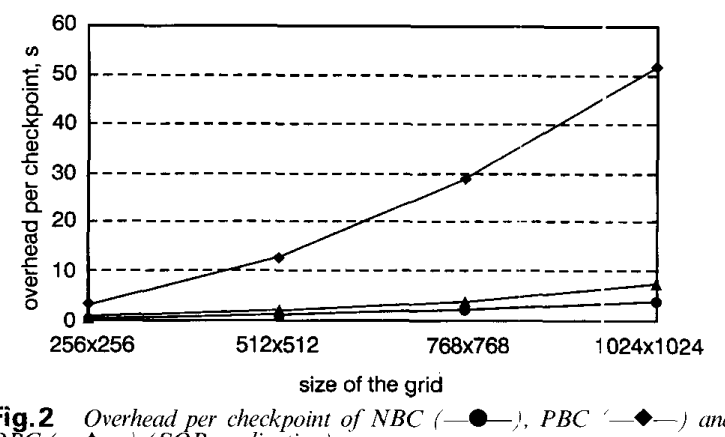
Fig.2 Overhead per checkpoint
$D B C(-\Lambda-)(S O R$ application $)$

Neighbour based checkpointing presents a lower overhead per checkpoint than PBC, but the DBC scheme incurs a comparable overhead. The overhead of the PBC technique is much higher than the other two schemes. Take, for instance, the case of a g id size of $1024 \times 1024$ : while the overhead per checkpinint introduced by NBC and DBC was 3.7 and 7.2 seconds, respectively, the overhead of $\mathrm{PBC}$ was around $52 \mathrm{sec}-$ onds. The main reason why $\mathrm{PBC}$ performs a lot worse 
than NBC is due to the bottleneck caused by the centralised parity operation. Processor 0 takes the role of the parity processor, but it also runs a process from the application. The execution of the PBC scheme leads to high congestion on this particular processor which results in a slower parity computation. This is the clear disadvantage of the centralised approach over the distributed approach taken by NBC.

Disk based checkpointing can have a similar overhead to NBC but presents a much higher checkpoint latency. However, the NBC scheme is not able to tolerate total failures, and in our particular implementation, it does not provide checkpoint atomicity if a failure occurs during the checkpointing protocol. To tolerate these types of failures we have to use this scheme together with checkpoints on disk. In the following Section, we present the performance results of a mixed scheme based on two-level stable storage, where we combine DBC with $\mathrm{NBC}$.

\subsection{Two-level stable storage}

One of the advantages of neighbour based checkpointing is the potential for taking checkpoints more frequently. We can see in Fig. 3 that the checkpoint overhead is in fact very small. It represents the total performance overhead introduced by the NBC scheme when executing the ISING application for about 4 hours. With an interval between checkpoints of more than 1 minute the overhead was always lower than $1 \%$. Reducing the interval to 30 and 10 seconds results in a natural increase in the total overhead to $2.2 \%$ and $6.7 \%$, respectively. Even with this very high frequency of checkpointing the resulting overhead is still acceptable. With the most conservative checkpointing interval $(10 \mathrm{~s})$ the total execution time with a grid of $1280 \times$ 1280 is increased by less than $7 \%$. If there is a processor failure, at most 10 seconds of computation are lost, which represents a very fast recovery.

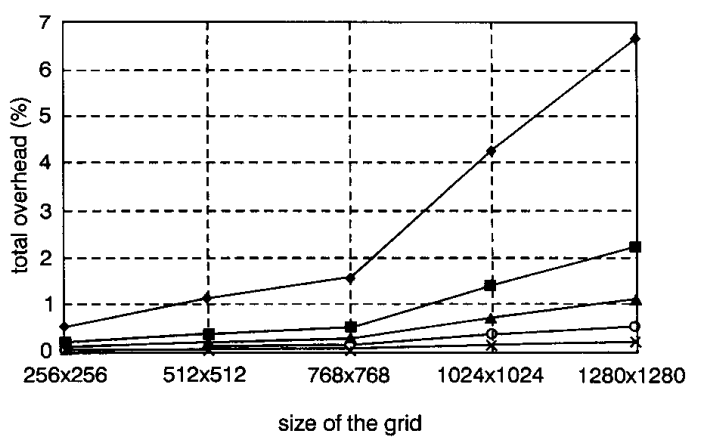

Fig.3 Total overhead of NBC with different checkpoint intervals ISING)

$-10 \mathrm{~s}-\square-30 \mathrm{~s}-\boldsymbol{\Delta}-1 \mathrm{~min}-\mathrm{O}-2 \mathrm{~min}-x-5 \mathrm{~min}$

However, if there is a total or multiple failure the application has to be restarted from scratch, rendering completely useless all the 'soft' checkpoints that have been taken so far. Thus, the best solution is to use this scheme (NBC) with disk based checkpointing (DBC): in the event of total/multiple failure, the application can be recovered from a 'hard' checkpoint kept on disk.

NBC can be used to checkpoint at short intervals while the DBC method is used to checkpoint at long intervals. In this way, a single processor failure can be restarted from a 'soft' checkpoint while all other failures can be overcome using previous 'hard' checkpoint.
As seen earlier, the overhead per checkpoint introduced by $\mathrm{DBC}$ is fairly close to the NBC overhead, although it takes a bit more time to complete a checkpoint. In Fig. 4 we present the total performance overhead when using two-level stable storage, that is, combined NBC and DBC. The interval between 'soft' checkpoints is termed $I B S C$ while the interval between 'hard' checkpoints is termed $I B H C$. Two values were chosen for IBSC: 10 and 30 seconds. With these two intervals we have chosen four possible combinations with DBC: no use of hard checkpoints, or the use of it with $I B H C$ equal to 1,2 and 5 minutes.

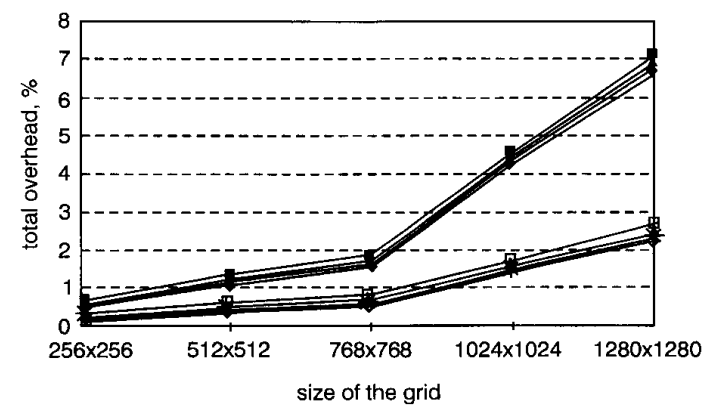

Fig.4 Total overhead of two-level stable storage (IBSC and IBHC) IBSC 10 s, no hard checkpoint IBSC $10 \mathrm{~s}$, no hard check
IBSC $10 \mathrm{~s}$, IBHC $1 \mathrm{~min}$ IBSC $10 \mathrm{~s}$, IBHC $2 \mathrm{~min}$ IBSC $30 \mathrm{~s}$, IBHC $5 \mathrm{~min}$ IBSC $30 \mathrm{~s}$, no hard check
IBSC $30 \mathrm{~s}$, IBHC $1 \mathrm{~min}$ IBSC $30 \mathrm{~s}$, IBHC $2 \mathrm{~min}$
IBSC $30 \mathrm{~s}$, IBHC $5 \mathrm{~min}$

It can clearly be seen that the total overhead is not significantly affected by the integration of 'hard' checkpoints with the NBC scheme. The maximum difference observed was $0.45 \%$, which is negligible. Thus, we can use this hybrid approach of NBC and DBC without additional cost. It is able to tolerate any number of failures, allows checkpoints to be taken more frequently and provides a faster recovery.

\section{Comparison with related work}

Parity checkpointing was first presented by Jim Plank in [12]. The first real implementation was reported in [16]. Parity checkpointing was later integrated in four subroutines of ScaLAPACK [17].

Recently, an implementation of neighbour based and parity checkpointing was reported [18]. These schemes were implemented in a different way to that used in our study: both schemes stop the application during the whole checkpoint operation and do not make use of any concurrent execution as in our case. Even so, it was shown that neighbour based checkpointing was an order of magnitude faster than parity checkpointing, but takes twice as much storage overhead.

An analytic model was presented in [19] to describe multilevel checkpoint storage schemes. A multilevel recovery scheme is one that can tolerate different numbers of failures at different costs, where the tolerance of a larger number of failures requires a larger overhead. A two-level scheme was then discussed and included 1checkpoints and N-checkpoints: 1-checkpoints are saved in volatile memory and thus present a smaller cost, albeit they tolerate only single process failures; $\mathrm{N}$-checkpoints are saved in stable storage and are able to tolerate multiple failures at the expense of a higher performance overhead. It was shown that to minimise 
the average overhead it may be advantageous to take both kinds of checkpoints. We have reached a similar conclusion based on an experimental study, rather than a probabilistic mathematical model.

\section{Conclusions}

In this study we have presented and evaluated two schemes for diskless checkpointing: parity and neighbour based checkpointing. While the parity based scheme introduces less memory overhead it incurs a higher performance overhead than the second technique. Another interesting result was that it is possible to implement disk based checkpointing with a similar overhead to neighbour based checkpointing, although with a higher checkpoint latency.

Although neighbour based checkpointing is a very efficient technique it does not provide a high level of reliability since it is not able to tolerate multiple and total failures. To increase the reliability of this scheme the best approach is to rely on two-level stable storage where neighbour based checkpointing (NBC) is integrated with disk based checkpointing (DBC). From time to time the application saves its state to a disk file ('hard' checkpoint). During that interval the application can checkpoint its state across the memory of the neighbour processors ('soft' checkpoint). This last scheme is efficient and provides fast recovery. If there is a partial failure in the system the application can be recovered from the 'soft' checkpoint. If there is a total failure the application is restarted from a checkpoint saved on disk. This approach of two-level stable storage combines the advantages of the two schemes: the efficiency of diskless checkpointing with the reliability of disk based checkpointing.

\section{Acknowledgments}

We would like to thank the anonymous referees for their thoughtful comments. This work was partially supported by the Portuguese Ministry of Science and Technology (MCT), the European Union through the R\&D Unit 326/94 (CISUC) and the project PRAXIS XXI 2/2.1/TIT/1625/95.

\section{References}

1 LAMPSON, B.W., and STURGIS, H.E.: 'Crash discovery in a distributed data storage system'. Technical Report XEROX Parc, April 1979

2 BARTLETT, J., GRAY, J., and HORST, B.: 'Fault tolerance in tandem computing systems' in 'Dependable computing; and faulttolerance systems' (Springer-Verlag, 1987)

3 JOHNSON, D.B.: 'Distributed system fault-tclerance using message logging and checkpointing'. $\mathrm{PhD}$ Thesis, TR-8 J-101, Rice University, Houston, Texas, December 1989

4 WILKES, J., and STATA, R.: 'Specifying data aviliability in multi-device file systems', Operating Syst. Rev., Janua y 1991, 25, (1), pp. 56-59

5 ELNOZAHY, E.N., JOHNSON, D.B., and Z.WAENEP OEL, W.: 'The performance of consistent checkpointing'. Proceedings of 11 th symposium on Reliable distributed sy:tems, 1992 pp. 3947

6 ELNOZAHY, E.N., and ZWAENEPOEL, W.: 'On the use and implementation of message logging'. Proceedings of 24th Fault tolerant computing symposium, FTCS-24, June 1994, rp. 298-307

7 PLANK, J.S., and LI, K.: 'ickp - A consistent check.pointer for multicomputers', IEEE Parallel Distrib. Technol., 199<, 2, (2), pp. $62-67$

8 SILVA, L.M.: 'Checkpointing mechanisms for scientific parallel applications'. PhD Thesis, University of Coimbra, March 1997

9 BANATRE, M.. MULLER, G., and BANATRE, J.P.: 'Ensuring data security and integrity with a fast stable storage'. Proceedings of 4 th conference on Data engineering, February 1988, pp. 285 293

10 HORN, C., COGHLAN, B., HARRIS, N., and JON ES, J.: 'Stable memory - another look'. International workshop on Operating systems of the 90's and beyond, 1991, pp. 171-177 (Lezture Notes on Computer Science, 563)

11 BAKER, M., and SULLIVAN, M.: 'The recovery bo:: using fast recovery to provide high availability in the UNIX environment' Proceedings of Summer'92 USENIX, June 1992, pp. 31-42

12 PLANK, J.S.: 'Efficient checkpointing on MIMD ar hitectures' PhD Thesis, Department of Computer Science, Prince ion University, June 1993

13 GIBSON, G.A.: 'Redundant disk arrays: reliable, parcllel second ary storage.' PhD Thesis, University of California it Berkeley, December 1990 14 Parix 1.2: Software documentation, Parsytec Computer $\mathrm{GmbH}$

15 MPI Forum, Message passing interface standard, March 1994 , available at: http:/www.netlib.org/mpi/

16 PLANK, J.S., and LI, K.: 'Faster checkpointing with N+1 parity'. Proceedings of 24th Fault-tolerant computing :yymposium, FTCS-24, June 1994, pp. 288-297

17 PLANK, J.S., KIM, Y., and DONGARRA, J.: "Algo ithm-based diskless checkpointing for fault-tolerant matrix conputations' Proceedings of 25th Fault-tolerant computing symposium, FTCS25 . June 1995 , pp. $351-360$

18 CHIUEH, T., and DENG, P.: 'Evaluation of checkpoint mechanisms for massively parallel machines'. Proceedings of 26th Faulttolerant computing symposium, FTCS-26, Japan, Jun? 1996, pp $370-379$

19 VAIDYA, N.H.: 'A case for multi-level distributed recovery schemes'. Technical Report 94-043, Dept. Computer Science, Texas A\&M University, 1994 